\title{
Cores of Simplicial Complexes
}

\author{
Mario Marietti · Damiano Testa
}

Received: 12 April 2007 / Revised: 7 April 2008 / Accepted: 16 April 2008 /

Published online: 15 May 2008

(C) Springer Science+Business Media, LLC 2008

\begin{abstract}
We introduce a method to reduce the study of the topology of a simplicial complex to that of a simpler one. Applying this method to complexes arising from graphs, we give topological meaning to classical graph invariants. As a consequence, we answer some questions raised in (Ehrenborg and Hetyei in Eur. J. Comb. 27(6):906-923, 2006) on the independence complex and the dominance complex of a forest and obtain improved algorithms to compute their homotopy types.
\end{abstract}

Keywords Simplicial complexes · Monomial ideals · Homotopy type · Independence and domination in forests

\section{Introduction}

In this paper we study the topology of a simplicial complex $\Delta$ by introducing a family $c(\Delta)$ of face links that we call the core of $\Delta$. The homotopy types of $\Delta$ and each element of its core are closely related. Indeed, we show that one of the following happens: either $\Delta$ collapses onto a point, or it is simple-homotopic to an iterated suspension of any element of its core.

The application motivating this method is in the study of the independence and dominance complexes of a graph, which have been extensively studied in the recent years $[6,11,12,18-21,27]$ because of their applications in graph theory, network analysis, and statistical mechanics. Our method allows us to obtain several results

M. Marietti

Università degli Studi di Roma "La Sapienza", Piazzale A. Moro 5, 00185 Roma, Italy

e-mail: marietti@mat.uniroma1.it

D. Testa ( $\square)$

Jacobs University Bremen, Campus Ring 1, 28759 Bremen, Germany

e-mail: d.testa@jacobs-university.de 
on the topology of the independence and dominance complexes. In particular, we give topological interpretations to some well-known graph invariants (e.g., the domination number, the independent domination number, the matching number, and the vertex covering number), which also have been an object of several studies (see, for instance, $[1,2,5,13,16,17])$. As a consequence, we answer some questions posed in [12]. Ehrenborg and Hetyei prove that the independence complex of a forest $F$ is always contractible or homotopic to a sphere and that the dominance complex of a forest $F$ is always homotopic to a sphere. Hence, they ask for a simple way to determine whether the independence complex of $F$ is contractible and, if not, to compute the dimension of the associated sphere, and similarly to compute the dimension of the sphere associated to the dominance complex of $F$. We use the cores to answer these questions, proving that the contractibility of the independence complex of $F$ is detected by some graph theoretical properties of $F$. When the independence complex of $F$ is contractible, it collapses onto a point; when the independence complex of $F$ is not contractible, it collapses onto the boundary of a cross-polytope whose dimension equals the domination number of $F$. Finally, we prove that the dominance complex of $F$ always collapses onto the boundary of a cross-polytope whose dimension equals the matching number of $F$.

The independent domination number and the matching number of a forest $F$ can be both computed by linear algorithms (see [8]). Hence the results in this work give an efficient way to compute the dimensions of the spheres associated to $F$ via both the independence and dominance complex.

For notational convenience, we use the terminology coming from commutative algebra (specifically monomial ideal theory).

The paper is organized as follows. Section 2 contains the notation and background needed in the sequel. In Sect. 3 we define the notion of domination between variables and study its relationship to suspension. In Sect. 4 we introduce and study the main new concept of this work, namely the core of a simplicial complex; we reduce the study of the topology of a simplicial complex to the study of its core. In Sect. 5 we apply the method of the core to the independence complex $\Delta$ of a forest $F$. We find several conditions which are equivalent to the contractibility of $\Delta$ and prove that, if $\Delta$ is not contractible, then it collapses onto the boundary of a cross-polytope whose dimension equals the domination number of $F$. In Sect. 6 we apply the method of the core to the dominance complex $\Delta$ of a forest $F$. We prove that $\Delta$ always collapses onto the boundary of a cross-polytope whose dimension equals the matching number of $F$.

\section{Notation and Background}

If $r \in \mathbb{Z}, r \geq 0$, we let $[r]:=\{1, \ldots, r\}$. The cardinality of a set $A$ is denoted by $|A|$.

We consider finite undirected graphs $G=(V, E)$ with no loops or multiple edges. For all $S \subset V$, let $N[S]:=\{w \in V \mid \exists s \in S,\{s, w\} \in E\} \cup S$ be the closed neighborhood of $S$; when $S=\{v\}$, then we let $N[v]=N[\{v\}]$. A set $D \subset V$ is called dominating if for all $v \in V, N[v] \cap D \neq \emptyset$. A set $D \subset V$ is called independent if no two vertices in $D$ are adjacent, i.e., $\left\{v, v^{\prime}\right\} \notin E$ for all $v, v^{\prime} \in D$. A vertex cover of $G$ 
is a subset $C \subset V$ such that every edge of $G$ contains a vertex of $C$. A matching of $G$ is a subset $M \subset E$ of pairwise disjoint edges.

We consider the following classical invariants of a graph $G$ which have been extensively studied by graph theorists (see, for instance, $[1,2,5,13,16,17]$ ); we let

- $\gamma(G):=\min \{|D|, D$ is a dominating set of $G\}$ be the domination number of $G$;

- $i(G):=\min \{|D|, D$ is an independent dominating set of $G\}$ be the independent domination number of $G$;

- $\alpha_{0}(G):=\min \{|C|, C$ is a vertex cover of $G\}$ be the vertex covering number of $G$;

- $\beta_{1}(G):=\max \{|M|, M$ is a matching of $G\}$ be the matching number of $G$.

Recall the following well-known result of König (cf. [10], Theorem 2.1.1).

Theorem 2.1 (König) Let $G$ be a bipartite graph. Then $\alpha_{0}(G)=\beta_{1}(G)$.

We refer the reader to [4] or [10] for all undefined notation on graph theory.

We let $X:=\left\{x_{1}, \ldots, x_{n}\right\}$ and $\mathbb{Z}[X]$ be the polynomial ring with variables $x_{1}, \ldots, x_{n}$ over the integers; we set $\mathbb{Z}[\emptyset]:=\mathbb{Z}$. Let $m, m^{\prime} \in \mathbb{Z}[X]$; we write $m^{\prime} \mid m$ if $m^{\prime}$ divides $m$.

Definition 2.2 A simplicial complex $\Delta$ on $X$ is a set of subsets of $X$, called faces, such that, if $\sigma \in \Delta$ and $\sigma^{\prime} \subset \sigma$, then $\sigma^{\prime} \in \Delta$. The faces of cardinality one are called vertices.

Equivalently, a simplicial complex $\Delta$ on $\mathbb{Z}[X]$ is a finite set of square-free monomials of $\mathbb{Z}[X]$ such that, if $m \in \Delta$ and $m^{\prime} \mid m$, then $m^{\prime} \in \Delta$.

We do not require that $\{x\} \in \Delta$ for all $x \in X$. We frequently identify a set $S \subset$ $X$ with the monomial $x_{1}^{\varepsilon_{1}} \cdots x_{n}^{\varepsilon_{n}}$, where $\varepsilon_{i}=\left\{\begin{array}{l}1 \text { if } x_{i} \in S ; \\ 0 \text { if } x_{i} \notin S\end{array}\right.$ Note that the empty set is identified with the monomial 1 . We refer the reader to [23] for all undefined concepts from commutative algebra.

Every simplicial complex $\Delta$ on $\mathbb{Z}[X]$ different from $\{1\}$ has a standard geometric realization. Let $e_{1}, \ldots, e_{n}$ be the standard basis of $\mathbb{R}^{n}$. The realization of $\Delta$ is the union of the convex hulls of the sets $\left\{e_{i}\right.$ such that $\left.x_{i} \mid m\right\}$ for each monomial $m \in \Delta$. Whenever we mention a topological property of $\Delta$, we implicitly refer to the geometric realization of $\Delta$ with the topology induced by $\mathbb{R}^{n}$.

Let $I \subset \mathbb{Z}[X]$ be a monomial ideal (i.e., an ideal generated by monomials) containing $x_{1}^{2}, \ldots, x_{n}^{2}$. The set of monomials of $\mathbb{Z}[X] \backslash I$ is a simplicial complex on $\mathbb{Z}[X]$ that we denote by $R(I)$. Conversely, given a simplicial complex $\Delta$ on $\mathbb{Z}[X]$, let $I_{\Delta} \subset \mathbb{Z}[X]$ be the ideal generated by the monomials not in $\Delta$. Clearly $\Delta=R\left(I_{\Delta}\right)$ and $I=I_{R(I)}$. Note that $I_{\Delta}$ is (essentially) the Stanley-Reisner ideal of the simplicial complex $\Delta$ (see [26]).

As examples, consider the ideals $I_{n}=\left(x_{1}^{2}, \ldots, x_{n}^{2}\right), J_{n}=\left(x_{1} \cdots x_{n}, x_{1}^{2}, \ldots, x_{n}^{2}\right)$, and $K_{n}=\left(x_{1} x_{2}, x_{3} x_{4}, \ldots, x_{2 n-1} x_{2 n}, x_{1}^{2}, \ldots, x_{2 n}^{2}\right)$. The simplicial complex $R\left(I_{n}\right)$ is the (n-1)-dimensional simplex, and $R\left(J_{n}\right)$ is its boundary; $R\left(K_{n}\right)$ is the boundary of the $n$-dimensional cross-polytope, which is the dual of the $n$-dimensional cube. Note that the cube, its boundary, and the cross-polytope are not simplicial complexes. Furthermore $R\left(K_{n}\right)$ is the $n$th suspension of the simplicial complex $\{1\}$. 
From now on, unless explicitly mentioned otherwise, $I$ denotes a monomial ideal of $\mathbb{Z}[X]$ containing $x_{1}^{2}, \ldots, x_{n}^{2}$, and $\Delta:=R(I)$.

For the basic concepts of simplicial homology, we refer the reader to [24]. We identify the free Abelian group of simplicial chains on $\Delta$ with the quotient $\mathbb{Z}[X] / I$ : the chains of dimension $i-1$ are the span of the monomials of degree $i$. Choose an order on $X$; this induces a boundary map $\delta$ on the simplicial chains. We denote by $Z(\Delta)$ the $\mathbb{Z}$-module of cycles on $\Delta$ and by $\tilde{\mathrm{H}}(\Delta, \mathbb{Z})$ the reduced homology groups with integer coefficients of $\Delta$. A quasi-isomorphism of degree $r$ is a morphism of chains sending chains of degree $k$ to chains of degree $k+r$ which induces an isomorphism in homology.

Let $M$ be a finitely generated graded $\mathbb{Z}[X]$-module $M$; we denote the (multigraded) Hilbert series of $M$ by $H\left(M ; x_{1}, \ldots, x_{n}\right)$. The multi-graded face polynomial $\mathcal{F}_{\Delta}\left(x_{1}, \ldots, x_{n}\right)$ of $\Delta$ is the polynomial of $\mathbb{Z}[X]$

$$
\mathcal{F}_{\Delta}\left(x_{1}, \ldots, x_{n}\right):=\sum_{m \in \Delta} m=H\left(\mathbb{Z}[X] / I ; x_{1}, \ldots, x_{n}\right) .
$$

The face polynomial $F_{\Delta}(t)$ of $\Delta$ is the polynomial

$$
F_{\Delta}(t):=\sum_{m \in \Delta} t^{\operatorname{deg} m}=\mathcal{F}_{\Delta}(t, \ldots, t) .
$$

The reduced Euler characteristic of $\Delta$ is $\tilde{e}(\Delta):=-F_{\Delta}(-1)$.

We note that the simplicial complexes $R\left(\left(x_{1}, \ldots, x_{n}\right)\right)=\{1\}$ and $R(\mathbb{Z}[X])=\emptyset$ are different: we call $\{1\}$ the $(-1)$-dimensional sphere, and $\emptyset$ the $(-1)$-dimensional simplex. The empty simplex $R(\mathbb{Z}[X])$ is contractible. For $n \geq 1$, let $S^{n-2}:=$ $R\left(\left(x_{1} \cdots x_{n}, x_{1}^{2}, \ldots, x_{n}^{2}\right)\right)$, the sphere of dimension $n-2$. Consistently with these conventions, the reduced Euler characteristic of the $(-1)$-dimensional sphere is -1 , while the reduced Euler characteristic of the $(-1)$-dimensional simplex is 0 .

Let $x \in \mathbb{Z}[X]$ be a monomial, and define the simplicial complexes

$$
\begin{aligned}
& (\Delta: x):=\{m \in \Delta \mid x m \in \Delta\}=R(I: x), \\
& (\Delta, x):=\{m \in \Delta \mid x \nmid m\}=R(I, x),
\end{aligned}
$$

where $(I: x)=\{m \in \mathbb{Z}[X] \mid x m \in I\}$, and $(I, x)$ is the ideal generated by $I$ and $x$. The simplicial complexes $(\Delta: x)$ and $(\Delta, x)$ are usually called respectively link and face-deletion of $x$. If $I_{1}, \ldots, I_{k} \subset \mathbb{Z}[X]$ are monomial ideals containing $x_{1}^{2}, \ldots, x_{n}^{2}$, then we define

$$
\operatorname{join}\left(R\left(I_{1}\right), \ldots, R\left(I_{k}\right)\right):=\left\{\operatorname{lcm}\left\{m_{i}, i \in[k]\right\} \mid m_{i} \in R\left(I_{i}\right)\right\}
$$

If $x$ and $y$ are monomials, let

$$
\begin{aligned}
A_{x}(\Delta) & :=\operatorname{join}(\Delta,\{1, x\}), \\
\Sigma_{x, y}(\Delta) & :=\operatorname{join}(\Delta,\{1, x, y\}) .
\end{aligned}
$$


If $x, y \in X$, then $A_{x}(\Delta)$ and $\Sigma_{x, y}(\Delta)$ are both simplicial complexes. If $x \neq$ $y \in X$ and they are coprime with the faces of $\Delta$, then $A_{x}(\Delta)$ and $\Sigma_{x, y}(\Delta)$ are called respectively the cone on $\Delta$ with apex $x$ and the suspension of $\Delta$. If $x \neq y$ and $x^{\prime} \neq y^{\prime}$ are variables in $X$ coprime with all the faces of $\Delta$, then the suspensions $\Sigma_{x, y}(\Delta)$ and $\Sigma_{x^{\prime}, y^{\prime}}(\Delta)$ are isomorphic; hence in this case sometimes we drop the subscript from the notation. It is well known that if $\Delta$ is contractible, then $\Sigma(\Delta)$ is contractible, and that if $\Delta$ is homotopic to a sphere of dimension $k$, then $\Sigma(\Delta)$ is homotopic to a sphere of dimension $k+1$.

We recall the notions of collapse and simple-homotopy (see [9]). Let $\sigma \supset \tau$ be faces of a simplicial complex $\Delta$, and suppose that $\sigma$ is maximal and $\operatorname{deg}(\tau)=$ $\operatorname{deg}(\sigma)-1$. If $\sigma$ is the only face of $\Delta$ properly containing $\tau$, then the removal of $\sigma$ and $\tau$ is called an elementary collapse. If a simplicial complex $\Delta^{\prime}$ is obtained from $\Delta$ by an elementary collapse, we write $\Delta \succ \Delta^{\prime}$.

Equivalently in terms of ideals, an elementary collapse is obtained by adding to the monomial ideal $I$ a monomial $\tau$ such that

- $\tau$ is a monomial not in $I$, and

- there is a unique variable $a$ such that $\sigma:=a \tau$ is also not in $I$.

When $\Delta^{\prime}$ is a subcomplex of $\Delta$, we say that $\Delta$ collapses onto $\Delta^{\prime}$ if there is a sequence of elementary collapses leading from $\Delta$ to $\Delta^{\prime}$.

Definition 2.3 Two simplicial complexes $\Delta$ and $\Delta^{\prime}$ are simple-homotopic if they are equivalent under the equivalence relation generated by $\succ$.

It is clear that if $\Delta$ and $\Delta^{\prime}$ are simple-homotopic, then they are also homotopic, and that a cone collapses onto a point.

Let $a \in X$. If $a \in I$, then $a$ is not a vertex of $\Delta$. Since we are interested in studying $\Delta$, we identify $I \subset \mathbb{Z}[X]$ with $J \subset \mathbb{Z}[X \backslash\{a\}]$ whenever $I=(J, a)$, because the associated simplicial complexes on $X$ and $X \backslash\{a\}$ are the same. Note that, in general, a monomial ideal $J$ has a unique minimal generating set $M$ consisting of monomials. If $J=I_{\Delta}$, then we let $M=B \cup\left\{x_{1}^{2}, \ldots, x_{n}^{2}\right\}$ with $B \cap\left\{x_{1}^{2}, \ldots, x_{n}^{2}\right\}=\emptyset$ and we call the elements of $B$ the minimal square-free generators of $I_{\Delta}$. It follows from the definitions that $(I: a)=(I, a)$ if and only if $\Delta$ is a cone with apex $a$; equivalently, $\Delta$ is a cone with apex $a$ if and only if $a$ divides no monomial of $B$.

We give the following easy result for further reference.

Lemma 2.4 Let $\Delta$ be a simplicial complex, and let $x \in \mathbb{Z}[X]$ be a monomial; then $\Delta=A_{x}(\Delta: x) \cup(\Delta, x)$.

Proof There is an exact sequence

$$
0 \rightarrow \mathbb{Z}[X] /(I: x) \stackrel{\cdot x}{\longrightarrow} \mathbb{Z}[X] / I \longrightarrow \mathbb{Z}[X] /(I, x) \rightarrow 0,
$$

and hence $\mathcal{F}_{\Delta}=x \mathcal{F}_{(\Delta: x)}+\mathcal{F}_{(\Delta, x)}$. On the other hand, the multi-graded face polynomial of $A_{x}(\Delta: x) \bigcup_{(\Delta: x)}(\Delta, x)$ is

$$
\mathcal{F}_{(\Delta: x)}+x \mathcal{F}_{(\Delta: x)}+\mathcal{F}_{(\Delta, x)}-\mathcal{F}_{(\Delta: x)} .
$$

Note that $x$ is coprime with all vertices of $(\Delta: x)$. 


\section{Domination}

In this section we introduce the notion of domination between variables in a monomial ideal $I \subset \mathbb{Z}[X]$ containing $x_{1}^{2}, \ldots, x_{n}^{2}$ and we give some preliminary results on the topology of the simplicial complex $R(I)$. The concept of domination is in fact purely combinatorial, since it uses the notion of collapse; a collapse is an instance of deformation retract, and hence the topological properties follow.

Since domination and suspension are closely related, we start with some remarks on suspensions of simplicial complexes. It is immediate that the suspension $\Sigma_{x, y} \Delta$ is a cone with apex $a$ if the simplicial complex $\Delta$ is a cone with apex $a$, independently of whether or not $x$ and $y$ are vertices of $\Delta$. On the contrary, if two simplicial complexes are simple-homotopic, it does not follow in general that their suspensions are homotopic. The next lemma analyses the question of lifting collapses to suspensions.

Lemma 3.1 Let $\Delta \succ \Delta^{\prime}$ be simplicial complexes, $\sigma \supset \tau$ the faces removed in the elementary collapse, and $x, y \in X, x \neq y$. Then the simplicial complex $\Sigma_{x, y}(\Delta)$ collapses onto $\Sigma_{x, y}\left(\Delta^{\prime}\right)$ unless one of the following is satisfied:

(1) $x \mid \tau, y \nmid \sigma$ and $y \frac{\sigma}{x} \notin \Delta^{\prime}, y \frac{\tau}{x} \in \Delta^{\prime}$;

(2) $y \mid \tau, x \nmid \sigma$ and $x \frac{\sigma}{y} \notin \Delta^{\prime}, x \frac{\tau}{y} \in \Delta^{\prime}$.

In these last cases the two suspensions have different Euler characteristics.

Proof For notational convenience, we may write $a$ for the singleton $\{a\}$.

Notice that $\Delta=\Delta^{\prime} \cup \tau \cup \sigma$ and hence

$$
\Sigma_{x, y}(\Delta)=\Sigma_{x, y}\left(\Delta^{\prime}\right) \cup \tau \cup \sigma \cup \operatorname{lcm}\{x, \tau\} \cup \operatorname{lcm}\{x, \sigma\} \cup \operatorname{lcm}\{y, \tau\} \cup \operatorname{lcm}\{y, \sigma\},
$$

where the unions need not be disjoint. We separate six mutually exclusive cases.

Case 1. $x, y \mid \sigma$. We show that $\Sigma_{x, y}(\Delta)=\Sigma_{x, y}\left(\Delta^{\prime}\right)$. By (3.1) it suffices to check that $\sigma \in \Sigma_{x, y}\left(\Delta^{\prime}\right)$. By hypothesis there is $t \in\{x, y\}$ such that $t \mid \tau$; then $\frac{\sigma}{t} \in \Delta^{\prime}$ and hence $\sigma \in \Sigma_{x, y}\left(\Delta^{\prime}\right)$.

Case 2. $x, y \nmid \sigma$. The union (3.1) is disjoint, and $\Sigma_{x, y}\left(\Delta^{\prime}\right)$ is obtained from $\Sigma_{x, y}(\Delta)$ by the elementary collapses of the faces $x \sigma \supset x \tau$ and $y \sigma \supset y \tau$ (in any order), followed by the collapse of the faces $\sigma \supset \tau$.

Case 3. $\sigma=x \tau, y \nmid \sigma$. By (3.1) we have $\Sigma_{x, y}(\Delta)=\Sigma_{x, y}\left(\Delta^{\prime}\right) \cup \tau \cup \sigma \cup y \tau \cup y \sigma$; collapsing successively the faces $y \sigma \supset y \tau$ and $\sigma \supset \tau$, we conclude.

Case 4. $\sigma=y \tau, x \nmid \sigma$. Follows by symmetry from Case 3.

Case 5. $x \mid \tau, y \nmid \sigma$. Note that $\frac{\sigma}{x} \in \Delta^{\prime}$ and hence $\sigma \in \Sigma_{x, y}\left(\Delta^{\prime}\right)$. Thus by (3.1) we have $\Sigma_{x, y}(\Delta)=\Sigma_{x, y}\left(\Delta^{\prime}\right) \cup y \tau \cup y \sigma$. We have

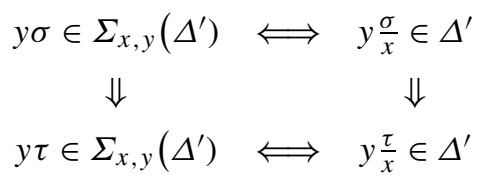

and hence 
Fig. 1 The simplicial complexes $R(I)$ and $R(J)$

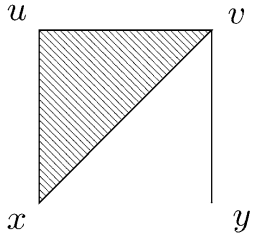

$R(I)$

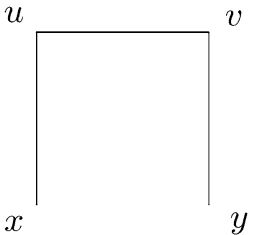

$R(J)$

- if $y \frac{\sigma}{x} \in \Delta^{\prime}$, then $\Sigma_{x, y}(\Delta)=\Sigma_{x, y}\left(\Delta^{\prime}\right)$;

- if $y \frac{\tau}{x} \notin \Delta^{\prime}$, then $\Sigma_{x, y}\left(\Delta^{\prime}\right)$ is obtained by the elementary collapse of the faces $y \sigma \supset y \tau$ of $\Sigma_{x, y}(\Delta)$;

- if $y \frac{\sigma}{x} \notin \Delta^{\prime}$ and $y \frac{\tau}{x} \in \Delta^{\prime}$, then (3.1) is satisfied. Note that $\Sigma_{x, y}(\Delta)=\Sigma_{x, y}\left(\Delta^{\prime}\right) \cup$ $y \sigma$; thus the Euler characteristics of $\Sigma_{x, y}(\Delta)$ and $\Sigma_{x, y}\left(\Delta^{\prime}\right)$ differ by one.

Case 6. $y \mid \tau, x \nmid \sigma$. Follows by symmetry from Case 5 .

Remark 3.2 Note that if at least one among $x$ or $y$ is not a vertex of $\Delta^{\prime}$, then $\Sigma_{x, y}(\Delta)$ collapses onto $\Sigma_{x, y}\left(\Delta^{\prime}\right)$, since (1) and (2) cannot be satisfied.

Example 3.3 Consider the following ideals of $\mathbb{Z}[x, y, u, v]$ :

- $I=\left(x y, y u, x^{2}, y^{2}, u^{2}, v^{2}\right)$,

- $J=\left(x y, y u, x v, x^{2}, y^{2}, u^{2}, v^{2}\right)$.

The complex $R(I)$ collapses onto $R(J)$ by the elementary collapse of the faces $x u v \supset x v$. On the other hand, $\Sigma_{x, y}(R(I))$ is the three-dimensional simplex with vertices $x, y, u, v$, while $\Sigma_{x, y}(R(J))$ is its boundary. This is case (1) of Lemma 3.1.

We now give the main definition of this section.

Definition 3.4 Let $a, b \in X$; $a$ dominates $b$ in $I$ if $R(I)$ is not a cone with apex $b$ and $R(I, a)$ is a cone with apex $b$.

Note that $a$ dominates $b$ in $I$ if and only if every minimal square-free generator of $I$ divisible by $b$ is also divisible by $a$ and there are such monomials. Loosely speaking, if $a$ dominates $b$, then $R(I)$ is composed out of two cones: the cone $R(I, a)$ with apex $b$ and the cone with apex $a$ on the subcomplex $R(I: a) \subset R(I, a)$ (see Fig. 2). The apex $a$ is not a vertex of $R(I, a)$, while $b$ might be a vertex of $R(I: a)$.

An immediate consequence of Lemma 2.4 is that, if $a, b \in X$ and $a$ dominates $b$ in $I$, then $R(I)$ is homotopic to $\Sigma(R(I: a))$. In fact we can prove that $R(I)$ is simple-homotopic to $\Sigma(R(I: a))$.

Theorem 3.5 Let a dominate $b$ in $I$, and let $I^{\prime}=\left(I, a b^{\prime},\left(b^{\prime}\right)^{2}\right) \subset \mathbb{Z}[X]\left[b^{\prime}\right]$. Then

(1) $R\left(I^{\prime}\right)$ collapses onto $R(I)$,

(2) $R(I)$ collapses onto $\Sigma_{a, b}(R(I: a))$,

(3) $R\left(I^{\prime}\right)$ collapses onto $\Sigma_{a, b^{\prime}}\left(R\left(I^{\prime}: a\right)\right)$. 
Fig. 2 The variable $a$ dominates $b$

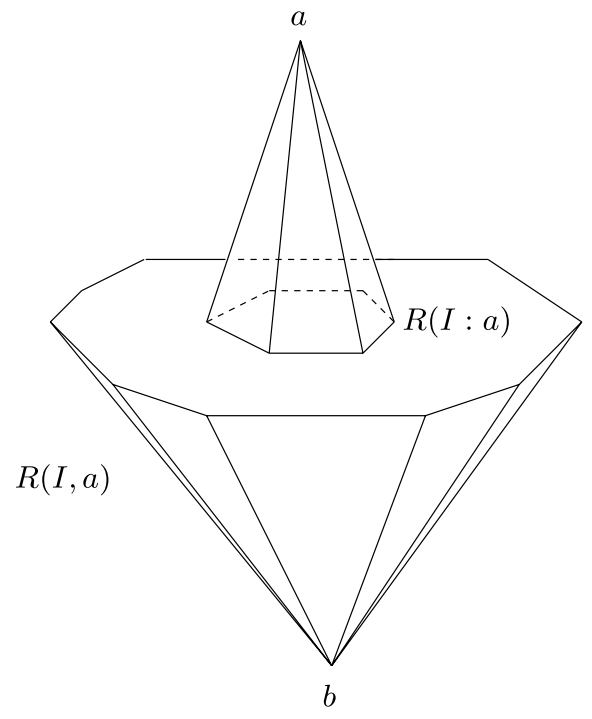

In particular, $R(I)$ is simple-homotopic to $\Sigma(R(I: a))$.

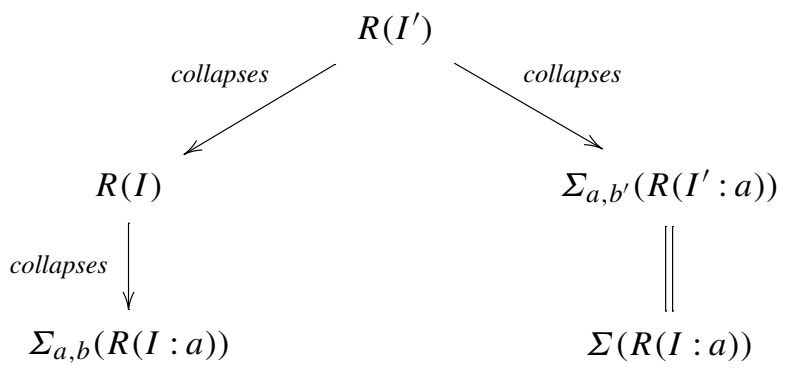

Proof (1) By definition $R(I) \subset R\left(I^{\prime}\right)$. We show that there is a sequence $\Delta_{0}:=$ $R\left(I^{\prime}\right) \succ \Delta_{1} \succ \cdots \succ \Delta_{s}:=R(I)$ of simplicial complexes, where $s$ is the number of faces of $R\left(I^{\prime}\right)$ containing $b$ and $b^{\prime}$, and $\Delta_{i}$ is obtained from $\Delta_{i-1}$ by the elementary collapse of a face $\sigma$ containing $b$ and $b^{\prime}$ and the face $\tau=\frac{\sigma}{b}$. Let $\Delta_{0}:=R\left(I^{\prime}\right)$; suppose that $\Delta_{j}$ has been defined for all $j \leq i \leq s$.

If $i=s$, then $b b^{\prime}$ is not a face of $\Delta_{i}$, since at each step we remove exactly one face containing $b b^{\prime}$. In this case we are done, since we have already removed the faces $\sigma=b b^{\prime}$ and $\frac{\sigma}{b}=b^{\prime}$, and thus $\Delta_{s}=R(I)$.

If $i<s$, we define $\Delta_{i+1}$ as follows. Note that $b b^{\prime}$ is a face of $\Delta_{i}$, since we removed fewer than $s$ faces containing $b b^{\prime}$, and let $f b b^{\prime}$ be a maximal face of $\Delta_{i}$ containing $b b^{\prime}$. We prove first that the only face strictly containing $f b^{\prime}$ is $f b b^{\prime}$. Let $q f b^{\prime}$ be a maximal face of $\Delta_{i}$ containing $f b^{\prime}$, and assume by contradiction that $q f b^{\prime} \neq f b b^{\prime}$. Clearly $a$ does not divide $q f$, since $a b^{\prime} \in I^{\prime}$, and $b$ cannot divide $q$, since $f b b^{\prime}$ is maximal. By the assumption on the elementary collapses, the faces of $R\left(I^{\prime}\right)$ not containing $b^{\prime}$ are not affected by the collapses. Hence $q f b$ is a face of $R(I, a)$, since $a$ dominates $b$ and $a \nmid q f$, and it is also a face of $\Delta_{j}$ for all $j \leq i$; the monomial $q f b b^{\prime}$ 
is a face of $\Delta_{0}$ and not of $\Delta_{i}$ by maximality. Hence there is an index $j<i$ such that $\Delta_{j+1}$ is obtained by removing the faces $\sigma=q f b b^{\prime}$ and $\frac{\sigma}{b}$, contradicting the fact that $q f b^{\prime} \in \Delta_{i}$.

By what we just proved, we may collapse the faces $q f b b^{\prime}$ and $q f b^{\prime}$. We define $\Delta_{i+1}$ to be the result of this collapse. Iterating this procedure, we conclude.

(2) The simplicial complex $\Sigma_{a, b}(R(I: a))$ is a subcomplex of $R(I)$. Let $\sigma$ be a maximal face of $R(I)$ not in $\Sigma_{a, b}(R(I: a))$; we show that $\sigma$ contains $b$ and that we may collapse $\sigma$ and $\frac{\sigma}{b}$. Note that $\sigma$ does not contain $a$, since $\sigma$ is not a face of $\Sigma_{a, b}(R(I: a))$, and hence $\sigma$ is a face of $R(I, a)$; since $R(I, a)$ is a cone of apex $b$ and $\sigma$ is maximal, $\sigma$ contains $b$. Write $\sigma=\tau b$; if $\tau a$ is a face of $R(I)$, then $\tau \in R(I: a)$ and hence $\tau b \in \Sigma_{a, b}(R(I: a))$. Since this is not the case, if $\tau h$ is a face of $R(I)$ containing $\tau$, then $h$ is not divisible by $a$ and hence $\tau h$ is a face in $R(I, a)$; thus $\operatorname{lcm}\{\tau h, b\}$ is also a face of $R(I, a)$, and by maximality of $\sigma=\tau b$ we conclude that $h \mid b$. Thus the only face of $R(I)$ strictly containing $\tau$ is $\sigma$. Hence we may collapse the faces $\sigma$ and $\tau$ to obtain a simplicial complex $\Delta^{\prime}$. Note that $\left(\Delta^{\prime}, a\right)$ is again a cone with apex $b$, and we may iterate this procedure and conclude.

(3) Follows from part (2), since $a$ dominates $b^{\prime}$ in $I^{\prime}$.

The last statement follows since $\Sigma_{a, b^{\prime}}\left(R\left(I^{\prime}: a\right)\right)$ is isomorphic to the abstract suspension $\Sigma(R(I: a))$, because $a, b^{\prime} \notin R\left(I^{\prime}: a\right)=R(I: a)$.

Example 3.6 Let $I=\left(x_{1} x_{2} x_{3}, x_{1}^{2}, x_{2}^{2}, x_{3}^{2}\right)$ and $J=\left(x_{1} x_{2}, x_{3} x_{4}, x_{1}^{2}, x_{2}^{2}, x_{3}^{2}, x_{4}^{2}\right)$. We have that $x_{3}$ dominates $x_{1}$ in $I$ and $x_{3}$ dominates $x_{4}$ in $J$; moreover, $R\left(I: x_{3}\right)=$ $R\left(J: x_{3}\right)$ is the simplicial complex consisting of the two points $x_{1}, x_{2}$. Hence both $R(I)$ and $R(J)$ are simple-homotopic to the boundary of the 2-dimensional crosspolytope (a square) by Theorem 3.5. Whereas the simplicial complex $R(J)$ is actually the boundary of the 2-dimensional cross-polytope, the simplicial complex $R(I)$ is the boundary of the 2-dimensional simplex (a triangle).

The following lemmas are needed in the next section.

Lemma 3.7 Let $\Delta \succ \Delta^{\prime}$ be simplicial complexes, and let $\sigma \supset \tau$ be the faces removed in the elementary collapse. Then

(1) any cycle of $\Delta$ is a combination of faces different from $\sigma$;

(2) any cycle of $\Delta$ is homologous to a combination of faces different from $\tau$;

(3) the inclusion $\Delta^{\prime} \subset \Delta$ induces an isomorphism in homology.

Proof Write $\sigma=a \tau$ and choose an order of the variables such that $a$ is first.

(1) Let $z=c \sigma+\sum_{A \neq \sigma} c_{A} A$ be a cycle. Since

$$
0=\partial z=c \tau-c a \partial \tau+\sum_{A \neq \sigma} c_{A} \partial A,
$$

we have $c=0$, because the face $\tau$ is properly contained only in $\sigma$.

(2) Let $z=d \tau+\sum_{A \neq \tau} c_{A} A$ and note that $z-\partial(d \sigma)$ has the required property.

(3) Let $z=\partial\left(c \sigma+d \tau+\sum_{A \neq \sigma, \tau} c_{A} A\right)$. By part (2) we may assume that the coefficient of $\tau$ in $z$ is zero and hence that $c=0$. Then $z$ is the boundary of $-d \partial \sigma+d \tau+\sum_{A \neq \sigma, \tau} c_{A} A$, and we are done. 
Remark 3.8 If $\Delta^{\prime}$ is obtained from $\Delta$ by a sequence of elementary collapses, then the inclusion $\Delta^{\prime} \subset \Delta$ induces an isomorphism in homology.

Lemma 3.9 Let a dominate $b$ in $I$, and let $I^{\prime}=\left(I, a b^{\prime},\left(b^{\prime}\right)^{2}\right) \subset \mathbb{Z}[X]\left[b^{\prime}\right]$. Then the inclusion $R(I) \subset R\left(I^{\prime}\right)$ induces an isomorphism in homology whose inverse is induced by the map $\bar{\varphi}$ of chains given by

$$
m \longmapsto \begin{cases}m & \text { if } m \text { does not contain } b^{\prime}, \\ b \frac{m}{b^{\prime}} & \text { if } m \text { contains } b^{\prime} \text { and does not contain } b, \\ 0 & \text { if } m \text { contains } b b^{\prime},\end{cases}
$$

where $m$ is any face of $R\left(I^{\prime}\right)$, with the variables ordered so that $a<b^{\prime}<b<x$ for all remaining variables $x$.

Proof First of all, we check that $\bar{\varphi}$ is a map of chains. Let $m \in R\left(I^{\prime}\right)$ be a face; we only need to consider the case $m=b^{\prime} m^{\prime}$ with $m^{\prime}$ not containing $b$. In this case, $m^{\prime}$ cannot contain $a$, since $a b^{\prime}$ is not a face of $R\left(I^{\prime}\right)$, and, because $a$ dominates $b$ in $I$, it follows that $b m^{\prime}$ is a face of $R(I)$, as needed. Let $\bar{\imath}$ be the map of chains induced by the inclusion $R(I) \subset R\left(I^{\prime}\right)$, and let $z$ be a cycle in $R\left(I^{\prime}\right)$. To conclude it is enough to check that $z-\bar{\imath}(\bar{\varphi}(z))$ is a boundary in $R\left(I^{\prime}\right)$. We may write $z=b^{\prime} b A+b^{\prime} B+C$, where the chains in $A, B$ do not contain $b, b^{\prime}$, and the chains in $C$ do not contain $b^{\prime}$; note that the chains in $A, B$ cannot contain $a$ since $a b^{\prime}$ is not a face. Since $z$ is a cycle, we have

$$
0=\partial z=b A-b^{\prime} A+b^{\prime} b \partial A+B-b^{\prime} \partial B+\partial C,
$$

which implies that $-(A+\partial B)=0$ since it is the coefficient of the faces containing $b^{\prime}$ and not containing $b$. Hence we may write $z-\bar{\imath}(\bar{\varphi}(z))=b^{\prime} b A+\left(b^{\prime}-b\right) B=\partial\left(b^{\prime} b B\right)$. Note that $b^{\prime} b B$ is a chain since the faces in $B$ do not contain $a$ and $b B$ is a chain.

We note that applying $\bar{\varphi}$ to a chain simply deletes all terms containing the face $b b^{\prime}$ and replaces $b^{\prime}$ by $b$ in all remaining terms.

\section{Resolutions and Cores}

In this section we introduce the notions of resolution and core of a monomial ideal $I \subset \mathbb{Z}[X]$ containing $x_{1}^{2}, \ldots, x_{n}^{2}$, and we deduce topological properties of the simplicial complex $R(I)$ from the resolution and the core of $I$.

Let $\left(a_{1}, \ldots, a_{r}\right)$ be a sequence of variables of $\mathbb{Z}[X]$ and, for $i \in[r+1]$, let $I_{i}:=$ $\left(I: a_{1} \cdots a_{i-1}\right)$.

Definition 4.1 A resolution of $I$ is a sequence $A=\left(a_{1}, \ldots, a_{r}\right)$ such that, for all $i \in[r]$, either $R\left(I_{i}\right)$ is a cone with apex $a_{i}$, or there exists $b_{i} \in X$ such that $a_{i}$ dominates $b_{i}$ in $I_{i}$. 
We call $c(A):=I_{r+1}$ the core of $A, d(A):=r$ the depth of $A$, and

$$
\begin{aligned}
c(I) & :=\{c(A) \mid A \text { is maximal }\}, \\
d(I) & :=\min \{d(A) \mid A \text { is maximal }\}
\end{aligned}
$$

respectively the core and the depth of $I$.

The resolution $A$ is spherical if the simplicial complex $R\left(I_{i}\right)$ is not a cone of apex $a_{i}$ for all $i \in[r]$. The ideal $I$ is spherical if it admits a maximal resolution which is spherical, conical if it admits a resolution which is not spherical, and simple if $\left(x_{1}, \ldots, x_{n}\right) \in c(I)$.

Remark 4.2 We shall see that the properties of being spherical and conical are mutually exclusive (cf. Theorem 4.10).

Note that if $R(I)$ is a cone with apex $b$ and $a \neq b$, then $R(I: a)$ is a cone with apex $b$. Hence if $A$ is a resolution of $I$ and $R(c(A))$ is a cone with apex $b$, then all maximal resolutions extending $A$ must contain $b$.

Theorem 4.3 Let $A=\left(a_{1}, \ldots, a_{r}\right)$ be a resolution of $I$.

- If $A$ is conical, then $R(I)$ collapses onto a point.

- If $A$ is spherical and $a_{i}$ dominates $b_{i}$ in $\left(I: a_{1} \cdots a_{i-1}\right)$, then $R(I)$ collapses onto join $(\Sigma, R(c(A)))$, where $\Sigma:=$ join $\left(\left\{1, a_{1}, b_{1}\right\}, \ldots,\left\{1, a_{r}, b_{r}\right\}\right)$. In particular, $R(I)$ is simple-homotopic to $\Sigma^{d(A)}(R(c(A)))$.

Proof If $R(I)$ is a cone of apex $a_{1}$, then it collapses onto the point $a_{1}$. Otherwise by Theorem 3.5 the simplicial complex $R(I)$ collapses onto $\Sigma_{a_{1}, b_{1}} R\left(I: a_{1}\right)$.

Suppose first that $A$ is spherical and proceed by induction on $r$. By Theorem 3.5 part (2), the simplicial complex $R(I)$ collapses onto $\Sigma_{a_{1}, b_{1}} R\left(I: a_{1}\right)$. If $r=1$, we are done. Suppose $r \geq 2$. Let $A^{\prime}=\left(a_{2}, \ldots, a_{r}\right)$ and $\Sigma^{\prime}:=$ join $\left(\left\{1, a_{2}, b_{2}\right\}, \ldots\right.$, $\left.\left\{1, a_{r}, b_{r}\right\}\right)$. Note that $c\left(A^{\prime}\right)=c(A)$. To conclude, it suffices to show that $\Sigma_{a_{1}, b_{1}} R\left(I: a_{1}\right)$ collapses onto $\Sigma_{a_{1}, b_{1}}$ join $\left(\Sigma^{\prime}, R\left(c\left(A^{\prime}\right)\right)\right)=$ join $(\Sigma, R(c(A)))$.

By induction, $R\left(I: a_{1}\right)$ collapses onto join $\left(\Sigma^{\prime}, R\left(c\left(A^{\prime}\right)\right)\right)$, since $A^{\prime}$ is a spherical resolution of $\left(I: a_{1}\right)$. Since $a_{1}$ is not a vertex of $R\left(I: a_{1}\right)$, we apply repeatedly Lemma 3.1 (see Remark 3.2) to conclude.

Suppose now that $A$ is conical. Let $i$ be the smallest index such that $R(I$ : $\left.a_{1} \cdots a_{i-1}\right)$ is a cone with apex $a_{i}$. By what we just proved, $R(I)$ collapses onto $C:=\Sigma_{a_{1}, b_{1}} \cdots \Sigma_{a_{1-1}, b_{i-1}} R\left(I: a_{1} \cdots a_{i-1}\right)$. Since $C$ is an iterated suspension of a cone, it is a cone; thus $C$ and hence $R(I)$ collapse to a point.

Remark 4.4 Let $a_{1}, \ldots, a_{r}$ be distinct variables, and let $b_{1}, \ldots, b_{r}$ be variables such that $a_{j} \neq b_{i}$ for all $j \leq i$. Hence

$$
r+1 \leq s:=\left|\left\{a_{1}, \ldots, a_{r}, b_{1}, \ldots, b_{r}\right\}\right| \leq 2 r .
$$

Let $\Sigma:=\Sigma_{a_{1}, b_{1}} \cdots \Sigma_{a_{r}, b_{r}}(\{1\})$. If $s=2 r$, then $\Sigma$ is the boundary of the $r$ dimensional cross-polytope. If $s=r+1$, then $\Sigma$ is the boundary of the $r$-dimensional 
simplex. In the cases where $r+1<s<2 r$, the complex $\Sigma$ is a hybrid of the two extreme cases. All the complexes thus obtained are simple-homotopic.

The first case where $\Sigma$ may be different from the boundary of a simplex or a cross-polytope is for $r=3$. Let $I=\left(x_{1} x_{2}, x_{3} x_{4} x_{5}, x_{1}^{2}, \ldots, x_{5}^{2}\right)$, and let $\left(a_{1}, a_{2}, a_{3}\right)=$ $\left(x_{1}, x_{3}, x_{4}\right)$ and $\left(b_{1}, b_{2}, b_{3}\right)=\left(x_{2}, x_{5}, x_{5}\right)$. We have that $a_{i}$ dominates $b_{i}$ in $(I$ : $\left.a_{1} \cdots a_{i-1}\right)$ and $s=5$; the simplicial complex $R(I)$ is the suspension of the boundary of a triangle.

The following result gives an explicit description of the homology of a simplicial complex $\Delta$ in terms of the homology of the core of a resolution of $\Delta$.

Theorem 4.5 Let $A=\left(a_{1}, \ldots, a_{r}\right)$ be a spherical resolution of $I$, and suppose that $a_{i}$ dominates $b_{i}$ in $I_{i}:=\left(I: a_{1} \cdots a_{i-1}\right)$. Then there is a quasi-isomorphism $\varphi: Z(R(c(A))) \rightarrow Z(R(I))$ of degree $r$.

Furthermore $\varphi=\pi \circ \varphi^{\prime}$, where $\pi$ is a map sending each face $\sigma$ to $\pm \sigma$ and

$$
\begin{aligned}
\varphi^{\prime}: Z(R(c(A))) & \longrightarrow Z(R(I)), \\
z & \longmapsto \prod\left(a_{i}-b_{i}\right) z
\end{aligned}
$$

(with the convention that all terms containing a square are zero).

For all orderings of the variables such that $a_{1}<b_{1} \leq a_{2}<b_{2} \leq \cdots \leq a_{r}<b_{r}<x$ for all remaining variables $x, \pi$ is the identity map.

Proof If $r=0$, let $\varphi=i d$. By induction on $r$ we reduce to the case $r=1$, since a composition of quasi-isomorphisms is a quasi-isomorphism and the degrees add. To simplify the notation, let $a=a_{1}$ and $b=b_{1}$. Choose an order of the variables such that $a, b$ are the first two variables and $a<b$.

Suppose first that $a b \in I$. Define $\varphi$ to be the multiplication by $(a-b)$. Since $\Sigma_{a, b}(R(I: a))$ is a subcomplex of $R(I), \varphi(z)$ is a chain, and we immediately see that it is a cycle. The fact that $\varphi$ is a quasi-isomorphism of degree one follows from the Mayer-Vietoris sequence associated to the decomposition $\Sigma_{a, b}(R(I: a))=$ $A_{a}(R(I: a)) \cup A_{b}(R(I: a))$ : in fact, $\tilde{\mathrm{H}}_{*}\left(A_{a}(R(I: a)), \mathbb{Z}\right) \oplus \tilde{\mathrm{H}}_{*}\left(A_{b}(R(I: a)), \mathbb{Z}\right)=$ $(0)$, and

$$
0 \longrightarrow \tilde{\mathrm{H}}_{*}\left(\Sigma_{a, b}(R(I: a)), \mathbb{Z}\right) \stackrel{\delta}{\longrightarrow} \tilde{\mathrm{H}}_{*-1}(R(I: a), \mathbb{Z}) \longrightarrow 0
$$

is exact. By Theorem 3.5 and Remark 3.8, the inclusion $\Sigma_{a, b}(R(I)) \subset R(I)$ induces an isomorphism $\iota$ in homology. We are done, since $\varphi$ induces in homology the composition $\iota \circ \delta^{-1}$.

If $a b \notin I$, consider the ideal $I^{\prime}=\left(I, a b^{\prime},\left(b^{\prime}\right)^{2}\right) \subset \mathbb{Z}[X]\left[b^{\prime}\right]$. By the previous case we know that the multiplication by $\left(a-b^{\prime}\right)$ induces an isomorphism of degree one between the homology of $R\left(I^{\prime}: a\right)$ and the homology of $\Sigma_{a, b^{\prime}}\left(R\left(I^{\prime}: a\right)\right)$. We have 
the following commutative diagram:

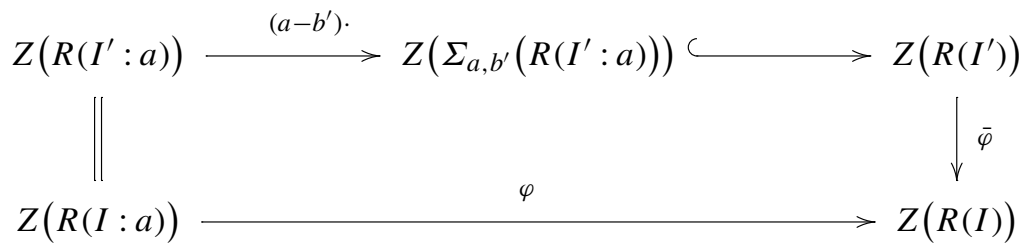

where $\bar{\varphi}$ is the map of Lemma 3.9. Since $\varphi$ is a composition of quasi-isomorphisms by the previous step and Lemmas 3.7 and $3.9, \varphi$ also is a quasi-isomorphism, and we are done.

We shall see that for the independence and dominance complexes of forests, the map $\pi$ of Theorem 4.5 always is the identity.

For the reader's convenience, we state explicitly the following easy results that will be used frequently in the sequel.

Lemma 4.6 Let $a_{1}, a_{2}, a_{3} \in \mathbb{Z}[X]$ be distinct variables.

(1) If $a_{1}$ dominates $a_{2}$ in $I$, then either $a_{1}$ dominates $a_{2}$ in $\left(I: a_{3}\right)$ or $R\left(I: a_{3}\right)$ is a cone with apex $a_{2}$.

(2) If $a_{1}$ dominates $a_{2}$ and $a_{2}$ dominates $a_{3}$ in I, then $a_{1}$ dominates $a_{3}$ in I.

(3) If $\left(a_{1}, a_{2}\right)$ is a resolution of $I, R\left(I: a_{1} a_{2}\right)$ is not a cone, and $a_{2}$ dominates $a_{3}$ in $I$, then $\left(a_{2}, a_{1}\right)$ also is a resolution of $I$.

Proof (1) Let $M$ (resp. $M^{\prime}$ ) be the set of minimal square-free generators of $I$ (resp. $\left.\left(I: a_{3}\right)\right)$ that are divisible by $a_{2}$; note that $M^{\prime} \subset M: a_{3}$. Since $a_{1}$ dominates $a_{2}$ in $I$, it follows that all monomials of $M$ are divisible also by $a_{1}$; because $a_{3} \neq a_{1}, a_{2}$, also all monomials of $M^{\prime}$ are divisible also by $a_{1}$. Moreover $M \neq \emptyset$. If $M^{\prime} \neq \emptyset$, then $a_{1}$ dominates $a_{2}$ in $\left(I: a_{3}\right)$; if $M^{\prime}=\emptyset$, then $R\left(I: a_{3}\right)$ is a cone with apex $a_{2}$.

(2) Since $a_{1}$ dominates $a_{2}$, every square-free minimal generator of $I$ divisible by $a_{2}$ is also divisible by $a_{1}$. Since $a_{2}$ dominates $a_{3}$, every square-free minimal generator of $I$ divisible by $a_{3}$ is also divisible by $a_{2}$ and hence by $a_{1} a_{2}$.

(3) If $a_{1}$ is the apex of a cone in $R(I)$, then everything is clear. Otherwise we may assume that $a_{1}$ dominates $b_{1}$ in $I$ with $b_{1} \neq a_{2}$ : indeed, if $a_{1}$ dominates $a_{2}$, since $a_{2}$ dominates $a_{3}$ in $I$, it follows that $a_{1}$ also dominates $a_{3} \neq a_{2}$ in $I$ by part (2). Applying part (1), either $a_{1}$ dominates $b_{1}$ in $\left(I: a_{2}\right)$, and we are done, or $R\left(I: a_{2}\right)$ is a cone with apex $b_{1}$, and hence also $R\left(I: a_{2} a_{1}\right)$ is a cone with apex $b_{1}$, contradicting the assumption.

The symmetric group on $X$ acts naturally on $\mathbb{Z}[X]$. This action need not preserve the ideal $I$, but in some cases it does. We denote by $\sigma_{x y}$ the transposition of the variables $x$ and $y$.

Lemma 4.7 Let $A=\left(a_{1}, \ldots, a_{r}\right)$ be a spherical resolution of $I$, and suppose that $R(c(A))$ is not a cone. Let $a \notin\left\{a_{1}, \ldots, a_{r}\right\}$, a dominate $b$ in $I$, and $I_{i}:=(I$ : $\left.a_{1} \cdots a_{i-1}\right)$ for $i \in[r]$. Then at least one of the following happens: 
(1) the sequence $\left(a_{1}, \ldots, a_{r}, a\right)$ is a spherical resolution;

(2) there is an index $i \in[r]$ such that a dominates $a_{i}$ in $I_{i}, a_{i}$ dominates $a$ in $I_{i}$, the sequence $A^{\prime}=\left(a_{1}, \ldots, a_{i-1}, a, a_{i+1}, \ldots, a_{r}\right)$ is a spherical resolution, and $c(A)=\sigma_{a a_{i}}\left(c\left(A^{\prime}\right)\right)$.

Proof For $i \in[r]$, let $a_{i}$ dominate $b_{i}$ in $I_{i}$. If $b \neq a_{1}$, then by Lemma 4.6 part (1) $a$ dominates $b$ in $I_{1}$, since $R\left(I_{1}\right)$ cannot be a cone, because $R(c(A))$ is not a cone. Thus, if $b \neq a_{i}$ for all $i \in[r]$, we prove that we are in case (1) by iterating this argument. Otherwise, suppose that there is an index $i$ such that $b=a_{i}$. Then $a_{i}$ dominates $b_{i}$ in $I_{i}$, and $a$ dominates $b=a_{i}$ in $I$ and hence in $I_{i}$ by Lemma 4.6 part (1). If $a \neq b_{i}$, by Lemma 4.6 part (2) $a$ dominates also $b_{i}$, we may replace $b$ by $b_{i}$ and reduce to the case $b \neq a_{i}$.

It remains to treat the case where $a=b_{i}$ and $b=a_{i}$ : in this case, $a$ and $a_{i}$ mutually dominate each other in $I_{i}$. Hence every square-free minimal generator of $I_{i}$ divisible by $a$ is divisible by $a_{i}$, and conversely. Thus exchanging $a$ and $a_{i}$ is an isomorphism of $\mathbb{Z}[X]$ that fixes $I_{i}$. Hence the sequence $\left(a_{1}, \ldots, a_{i-1}, a, a_{i+1}, \ldots, a_{r}\right)$ is a resolution of $I$. This is case (2).

Remark 4.8 The proof of Lemma 4.7 implies that if $b \notin\left\{a_{1}, \ldots, a_{r}\right\}$, then (1) certainly holds.

Corollary 4.9 Let $A=\left(a_{1}, \ldots, a_{r}\right)$ be a spherical resolution of $I$, and let a $\notin$ $\left\{a_{1}, \ldots, a_{r}\right\}$ dominate $b$ in I. Suppose that $R(c(A))$ is not a cone.

(1) If $b \notin\left\{a_{1}, \ldots, a_{r}\right\}$, then the sequence $\left(a_{1}, \ldots, a_{i}, a, a_{i+1}, \ldots, a_{r}\right)$ is a spherical resolution for $0 \leq i \leq r$.

(2) If $A$ is a maximal resolution, then there exists $i \in[r]$ such that a dominates $a_{i}$ in $I_{i}, a_{i}$ dominates $a$ in $I_{i}, A^{\prime}=\left(a, a_{1}, \ldots, a_{i-1}, a_{i+1}, \ldots, a_{r}\right)$ is a maximal resolution, and $c(A)=\sigma_{a a_{i}}\left(c\left(A^{\prime}\right)\right)$.

Proof (1) The result follows from Lemma 4.6 part (3) by induction on $r-i$, the case $r-i=0$ being Lemma 4.7 by Remark 4.8 .

(2) Since $A$ is maximal, we are in case (2) of Lemma 4.7. Thus there is an in$\operatorname{dex} i \in[r]$ such that $a$ dominates $a_{i}, a_{i}$ dominates $a$ in $I_{i}$, and the sequence $A^{\prime \prime}=\left(a_{1}, \ldots, a_{i-1}, a, a_{i+1}, \ldots, a_{r}\right)$ is a spherical resolution. From Lemma 4.7 it follows that $c(A)=\sigma_{a a_{i}}\left(c\left(A^{\prime \prime}\right)\right)$. Hence $A^{\prime \prime}$ is maximal. Otherwise, if $a^{\prime \prime}$ dominated $b^{\prime \prime}$ in $c\left(A^{\prime \prime}\right)$, then $\sigma_{a a_{i}}\left(a^{\prime \prime}\right)$ would dominate $\sigma_{a a_{i}}\left(b^{\prime \prime}\right)$ in $\sigma_{a a_{i}}\left(c\left(A^{\prime \prime}\right)\right)$. By applying repeatedly Lemma 4.6 part (3) we deduce that $A^{\prime}$ is also a maximal resolution and it spherical. Clearly $c\left(A^{\prime}\right)=c\left(A^{\prime \prime}\right)$.

We can now prove the main result of this section.

Theorem 4.10 Let I be a spherical ideal. Then all resolutions of I are spherical, all maximal resolutions of I have the same depth, and I has a unique core up to permutation of the variables. 
Proof Let $A=\left(a_{1}, \ldots, a_{r}\right)$ and $A^{\prime}=\left(a_{1}^{\prime}, \ldots, a_{s}^{\prime}\right)$ be maximal resolutions of $I$, and suppose that $A$ is spherical. We proceed by induction on $r$. If $r=0$, then the only maximal resolution of $I$ is the empty resolution; thus $I$ is its own core, and we are done.

Suppose that $r \geq 1$. Note first that $a_{1}^{\prime}$ is not the apex of a cone, since otherwise every maximal resolution of $I$ would contain $a_{1}^{\prime}$ and $I$ would not admit maximal resolutions that are spherical. If $a_{1}^{\prime} \notin\left\{a_{1}, \ldots, a_{r}\right\}$, then we may apply Lemma 4.7 to deduce that there is an index $i$ such that $a_{1}^{\prime}$ dominates $a_{i}$ and $\bar{A}=\left(a_{1}, \ldots, a_{i-1}, a_{1}^{\prime}, a_{i+1}, \ldots, a_{r}\right)$ is a spherical resolution. Moreover we have $c(A)=\sigma_{a_{1}^{\prime} a_{i}}(c(\bar{A}))$, and hence also $\bar{A}$ is maximal. Thus we may replace $A$ by $\bar{A}$ and assume that there is an index $i$ such that $a_{1}^{\prime}=a_{i}$. Applying Corollary 4.9, we may assume that $A=\left(a_{1}^{\prime}, a_{2}, \ldots, a_{r}\right)$, since changing the order of the elements of a maximal resolution does not affect the core of the resolution. In this case, we have that $\left(a_{2}, \ldots, a_{r}\right)$ and $\left(a_{2}^{\prime}, \ldots, a_{s}^{\prime}\right)$ are both maximal resolutions of $\left(I: a_{1}^{\prime}\right)$ and the first one is spherical. By induction we deduce that $r=s$ and that there exists a permutation $\sigma$ of the variables (different from $a_{1}^{\prime}$ ) such that

$$
c(A)=\left(\left(I: a_{1}^{\prime}\right): a_{2} \cdots a_{r}\right)=\sigma\left(\left(\left(I: a_{1}^{\prime}\right): a_{2}^{\prime} \cdots a_{r}^{\prime}\right)\right)=\sigma\left(c\left(A^{\prime}\right)\right)
$$

and $\left(a_{2}^{\prime}, \ldots, a_{r}^{\prime}\right)$ is a spherical resolution of $\left(I: a_{1}^{\prime}\right)$. Hence $A^{\prime}=\left(a_{1}^{\prime}, a_{2}^{\prime}, \ldots, a_{r}^{\prime}\right)$ is a spherical resolution of $I$, and the proof is complete.

Example 4.11 Consider the ideal $I \subset \mathbb{Z}\left[x_{1}, \ldots, x_{7}\right]$ generated by $x_{1}^{2}, \ldots, x_{7}^{2}$ and by the six monomials $x_{1} x_{2}, x_{3} x_{7}, x_{5} x_{6}, x_{5} x_{7}, x_{1} x_{3} x_{4}$, and $x_{2} x_{3} x_{4}$. The sequences $A_{1}=$ $\left(x_{5}, x_{4}\right)$ and $A_{2}=\left(x_{3}, x_{6}\right)$ are both maximal spherical resolutions of $I$. The cores are

$$
\begin{aligned}
& c\left(A_{1}\right)=\left(x_{4}, x_{5}, x_{6}, x_{7}, x_{1} x_{2}, x_{1} x_{3}, x_{2} x_{3}, x_{1}^{2}, x_{2}^{2}, x_{3}^{2}\right), \\
& c\left(A_{2}\right)=\left(x_{3}, x_{5}, x_{6}, x_{7}, x_{1} x_{2}, x_{1} x_{4}, x_{2} x_{4}, x_{1}^{2}, x_{2}^{2}, x_{4}^{2}\right),
\end{aligned}
$$

and $\sigma_{x_{3} x_{4}}\left(c\left(A_{1}\right)\right)=c\left(A_{2}\right)$.

\section{The Independence Complex}

In this section we apply the techniques developed in Sects. 3 and 4 to the independence complex of a forest.

Let $G=(V, E)$ be a graph with vertex set $V=\left\{x_{1}, \ldots, x_{n}\right\}$. Let $\bar{G} \subset \mathbb{Z}[X]$ be the ideal generated by $x_{1}^{2}, \ldots, x_{n}^{2}$ and by $x_{i} x_{j}$ for all $\left\{x_{i}, x_{j}\right\} \in E$. The ideal $\bar{G}$ is called the edge ideal of $G$, and the simplicial complex $R(\bar{G})$ is called the independence complex of $G$. The faces of the independence complex are the independent sets of $G$. In particular, if $n=0$, then $\bar{G}:=(0) \subset \mathbb{Z}$. We have

$$
\begin{aligned}
(\bar{G}, v) & =\overline{G \backslash\{v\}}, \\
(\bar{G}: v) & =\overline{G \backslash N[v]},
\end{aligned}
$$


where for all $S \subset V, G \backslash S$ is the graph obtained by removing from $G$ the vertices in $S$ and all the edges having a vertex in $S$ as an endpoint.

Let $a$ and $b$ be vertices of $G$. The simplicial complex $R(\bar{G})$ is a cone of apex $a$ if and only if $a$ is an isolated vertex of $G$. It follows from (5.1) that $a$ dominates $b$ in $\bar{G}$ if and only if $b$ is a leaf and $a$ is adjacent to $b$. Thus, using Theorem 4.3, we have proved the following:

Proposition 5.1 Let $F$ be a forest. Then $\bar{F}$ is simple, and either

- $\bar{F}$ is conical, and then $R(\bar{F})$ collapses onto a point, or

- $\bar{F}$ is spherical, and then $R(\bar{F})$ collapses onto the boundary of a cross-polytope of dimension $d(\bar{F})$.

Proof Since a forest always has either a leaf or an isolated vertex, the result follows from the discussion preceding the proposition.

The fact that $R(\bar{F})$ collapses either onto a point or onto the boundary of a crosspolytope can also be proved using Lemma 3.2 of [11].

The next corollary gives several characterizations of when $\bar{F}$ is conical and consequently when spherical.

Corollary 5.2 Let $F$ be a forest. The following are equivalent:

(1) the ideal $\bar{F}$ is conical;

(2) the complex $R(\bar{F})$ is contractible;

(3) the reduced Euler characteristic $\tilde{e}(\bar{F})$ is 0 ;

(4) the reduced Euler characteristic $\tilde{e}(\bar{F})$ is even;

(5) there is a sequence $\left(a_{1}, \ldots, a_{r}\right)$ of vertices such that $a_{i}$ is adjacent to a leaf of $F_{i}:=F \backslash N\left[\left\{a_{1}, \ldots, a_{i-1}\right\}\right]$ and $F_{r+1}$ contains an isolated vertex;

(6) for all maximal sequences $\left(a_{1}, \ldots, a_{r}\right)$ of vertices such that $a_{i}$ is adjacent to $a$ leaf of $F_{i}:=F \backslash N\left[\left\{a_{1}, \ldots, a_{i-1}\right\}\right]$, there is $i$ such that $F_{i}$ contains an isolated vertex;

(7) there is a vertex $v$ such that $R(\overline{F \backslash\{v\}})$ and $R(\overline{F \backslash N[v]})$ are either both contractible or both not contractible;

(8) for all vertices $v$, the complexes $R(\overline{F \backslash\{v\}})$ and $R(\overline{F \backslash N[v]})$ are either both contractible or both not contractible.

Proof The equivalence of (1)-(6) follows at once from Theorem 4.10 and Remark 4.2. By Lemma 2.4, we have

$$
\tilde{e}(\bar{F})=\tilde{e}(\bar{F}, v)-\tilde{e}(\bar{F}: v) .
$$

Thus $\tilde{e}(\bar{F})$ is even if and only if $\tilde{e}(\bar{F}, v) \equiv \tilde{e}(\bar{F}: v) \quad(\bmod 2)$. Since $(\bar{F}, v)=\overline{F \backslash\{v\}}$ and $(\bar{F}: v)=\overline{F \backslash N[v]}$, we may conclude using the equivalence of (2) and (4).

We now analyze the problem of computing the depth of $\bar{F}$. Hence, when $\bar{F}$ is spherical, we determine the dimension of the associated sphere. We prove that the depth of $\bar{F}$ equals the independent domination number of $F$. 
Fig. 3 The graph $F$

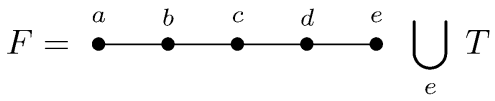

Fig. 4 The graph $F$

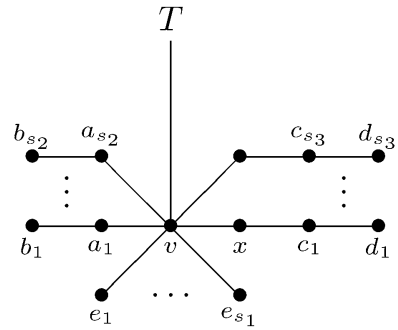

The dominating sets and the independent dominating sets of a graph have been studied by several graph theorists (see, for instance, $[5,16,22]$ ). The following lemma, which will be needed in the sequel, gives a result on the independent dominating sets of a forest.

Lemma 5.3 Let $F$ be a forest with at least one edge. There are independent dominating sets of $F$ of cardinality $i(F)$ containing a vertex at distance one from a leaf.

Proof We may assume that $F$ is a tree. Proceed by induction on the number of edges of $F$. If the number of edges of $F$ is at most three, then the result is clear.

Suppose that $F$ is as shown in Fig. 3, where $T$ is a tree containing the vertex $e$. Let $D$ be an independent dominating set of $F$ of cardinality $i(F)$. If $e \in D$, then by minimality $b \in D$, and we are done. If $e, d \notin D$, then necessarily $a, c \in D$. Hence also $(D \backslash\{a, c\}) \cup\{b, d\}$ is an independent dominating set of $F$ of cardinality $i(F)$ and we are done. Suppose finally that $e \notin D$ and $d \in D$. If $b \in D$, then we are done; otherwise $a$ must be in $D$, and we may replace $a$ by $b$.

The case of paths follows from what we said. Thus we assume that $F$ is not a path. Let $G$ be the smallest subtree of $F$ containing all vertices whose valence in $F$ is at least three, and let $v$ be a vertex whose valence in $G$ is at most one. Such a vertex exists, since $G$ is a tree and either it is a single vertex or it has at least one leaf. All components of the forest $F \backslash\{v\}$, except for at most one, are paths with an endpoint adjacent to $v$. By what we said above, we may assume that these paths consist of at most three vertices. For $i \in[3]$, we let $s_{i}$ be the number of such paths with $i$ vertices. Note that $s_{1}+s_{2}+s_{3} \geq 2$, since $v$ has valence at least three. For ease of presentation, we introduce the relevant notation in Fig. 4; the graph $T$ is a tree and may be empty. We consider two cases.

Case 1. There exists an independent dominating set $D$ of $F$ with $|D|=i(F)$ containing $v$. If $s_{1} \geq 1$, then we are done. If $s_{3} \geq 1$, then we are again done, since 
we may suppose that $D$ contains $c_{1}, \ldots, c_{s_{3}}$. Thus we assume that $s_{1}=s_{3}=0$, $s_{2} \geq 2$, and $b_{1}, \ldots, b_{s_{2}} \in D$. Let us consider the tree $F^{\prime}=\left(F \backslash\left\{b_{s_{2}}\right\}\right) \backslash\left\{a_{s_{2}}\right\}$. We have $i\left(F^{\prime}\right)=i(F)-1$. Indeed, $D \backslash\left\{b_{s_{2}}\right\}$ is an independent dominating set of $F^{\prime}$ of cardinality $i(F)-1$; conversely, if $D^{\prime}$ is an independent dominating set of $F^{\prime}$, then $D^{\prime} \cup\left\{b_{s_{2}}\right\}$ is an independent dominating set of $F$. By induction there exists an independent dominating set $D^{\prime \prime}$ of $F^{\prime}$ of cardinality $i\left(F^{\prime}\right)$ containing a vertex adjacent to a leaf $l$. The vertex $l$ is a leaf also in $F$, since the unique vertex of $F^{\prime}$ with a different valence in $F$ is $v$ and $v$ is not a leaf in $F^{\prime}$. Thus $D^{\prime \prime} \cup\left\{b_{s_{2}}\right\}$ is the required independent dominating set.

Case 2. Every independent dominating set of $F$ of cardinality $i(F)$ does not contain $v$. Let $D$ be an independent dominating set of $F$ with $|D|=i(F)$. If $s_{2} \geq 1$, then we may assume that $D$ contains $a_{1}, \ldots, a_{s_{2}}$, and we are done. If $s_{1} \geq 1$, then we may assume that $s_{3}=0$, since otherwise $D$ contains $c_{1}, \ldots, c_{s_{3}}$ by minimality. Thus either $s_{1} \geq 2$ and $s_{2}=s_{3}=0$, or $s_{1}=s_{2}=0$ and $s_{3} \geq 2$.

If $s_{1} \geq 2$ and $s_{2}=s_{3}=0$, then we consider the tree $F^{\prime}=F \backslash\left\{e_{s_{1}}\right\}$; by a similar reasoning as before we have $i\left(F^{\prime}\right)=i(F)-1$, and we conclude by the induction hypothesis.

Suppose finally that $s_{1}=s_{2}=0$ and $s_{3} \geq 2$. We may assume that $c_{1} \notin D$; hence $x, d_{1} \in D$, and by minimality $c_{2}, \ldots, c_{s_{3}} \in D$. This concludes the proof.

The following result gives a strict link between dominating sets of a forest $F$ and resolutions of the ideal $\bar{F}$.

Theorem 5.4 Let $F$ be a forest; then $i(F)=d(\bar{F})$. If $\bar{F}$ is spherical, then $\gamma(F)=$ $d(\bar{F})$.

Proof Let $\left(a_{1}, \ldots, a_{r}\right)$ be any maximal resolution; clearly $\left\{a_{1}, \ldots, a_{r}\right\}$ is an independent dominating set by (5.1), and we deduce that $i(F) \leq d(\bar{F})$. Thus we only need to prove that $i(F) \geq d(\bar{F})$.

Proceed by induction on $i(F)$, the base case being clear. If $E=\emptyset$, then the result is clear. So we may assume that $F$ has at least one edge. Let $D$ be an independent dominating set of minimum size. By Lemma 5.3 we may assume that $D$ contains a vertex $a_{1}$ at distance one from a leaf. We have $i\left(F \backslash N\left[a_{1}\right]\right)=i(F)-1$, since $D \backslash\left\{a_{1}\right\}$ is an independent dominating set of $F \backslash N\left[a_{1}\right]$, and if $D^{\prime}$ is an independent dominating set of $F \backslash N\left[a_{1}\right]$, then $D^{\prime} \cup\left\{a_{1}\right\}$ is an independent dominating set of $F$. Moreover $d(\bar{F}) \leq d\left(\bar{F}: a_{1}\right)+1$. By induction $d\left(\bar{F}: a_{1}\right) \leq i\left(F \backslash N\left[a_{1}\right]\right)$, and the first equality follows. Note that, in case $\bar{F}$ is spherical, we do not need to separate the two inequalities by Theorem 4.10.

To prove the second statement, we proceed by induction on the number of vertices of $F$. Since both $\gamma$ and $d$ are additive on connected components, we may assume that $F$ is a tree. If $F$ is a single edge, then the result is clear, since any dominating set of minimum size and any maximal resolution must contain one of the endpoints of the edge. Thus, without loss in generality, we only consider dominating sets of minimum size containing all vertices adjacent to a leaf. 
Suppose that $a$ dominates $b$ in $\bar{F}$ and that the distance of $b$ from the closest vertex of valence different from two is at least three. By Theorem 4.10, $a$ can be completed to a maximal resolution of depth $d(\bar{F})$, and hence we have

$$
\begin{aligned}
d(\bar{F}: a) & =d(\bar{F})-1, \\
\gamma(F \backslash N[a]) & =\gamma(F)-1,
\end{aligned}
$$

since any dominating set of $F$ must contain at least one of $a$ and $b$, and if it contains the other vertex adjacent to $a$, then we may simply "push it away" from $a$. By induction we have $d(\bar{F}: a)=\gamma(F \backslash N[a])$ and we conclude in this case.

Moreover, if $a$ dominates $b_{1}$ and $b_{2}, b_{1} \neq b_{2}$, then $d(\bar{F})=d\left(\overline{F \backslash\left\{b_{2}\right\}}\right)$ and $\gamma(F)=\gamma\left(F \backslash\left\{b_{2}\right\}\right)$, and we conclude by induction.

Thus we may assume that no vertex of $F$ dominates more than one vertex and that the distance of a leaf from a vertex of valence at least three is at most two. Since $\bar{F}$ is spherical, no vertex of $F$ has two leaves at distance one and two, respectively. Let $v$ be a leaf of the smallest tree containing all vertices of valence at least three of $F$. The forest $F \backslash\{v\}$ has at most one component which is not a path with an endpoint adjacent to $v$. With our reductions, all path components created by removing $v$ consist of exactly one edge:

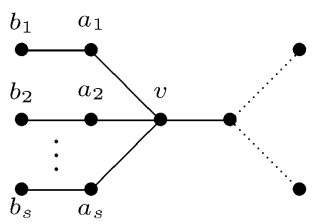

The graph $F$

We have $d(\bar{F})=d\left(\overline{F \backslash\left\{a_{s}, b_{s}\right\}}\right)+1$ and $\gamma(F)=\gamma\left(F \backslash\left\{a_{s}, b_{s}\right\}\right)+1$, and we conclude by induction.

Note that by Corollary 5.2 being conical or spherical can be defined in purely graph theoretic terms. As a consequence of Theorem 5.4, we have proved the following graph theoretic result.

Corollary 5.5 Let $F$ be a forest such that $\bar{F}$ is spherical. Then $\gamma(F)=i(F)$.

Remark 5.6 As shown in [3, 7, 8, 14], the domination and independent domination numbers of a forest can be computed by linear algorithms. Hence there are efficient ways to compute the dimension of the sphere associated to a forest $F$ such that $\bar{F}$ is spherical.

The problem of characterizing when the domination number equals the independent domination number appears in [22]; the forests for which this equality holds have been studied in [25]. 


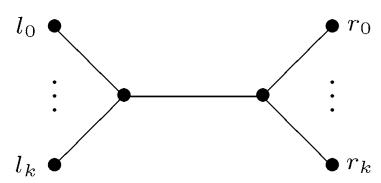

A tree $F$ with $i(F)-\gamma(F)=k$

The equality stated in Corollary 5.5 may be false if $\bar{F}$ is conical; indeed, the difference $i(F)-\gamma(F)$ can be any natural number.

Example 5.7 Consider the following tree $T$.

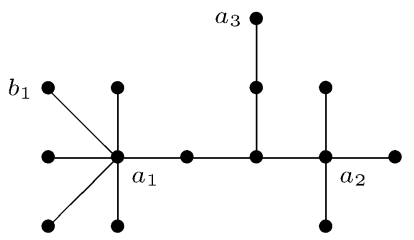

The tree $T$

Let us check that the sequence of vertices $\left(a_{1}, a_{2}, a_{3}\right)$ is a spherical resolution of $\bar{T}$. First of all, the vertex $a_{1}$ dominates $b_{1}$ (and each of the other four leaves adjacent to $\left.a_{1}\right)$. The simplicial complex $R\left(\bar{T}: a_{1}\right)$ is the same as the simplicial complex associated to the edge ideal of the graph $T_{1}=T \backslash N\left[a_{1}\right]$ :

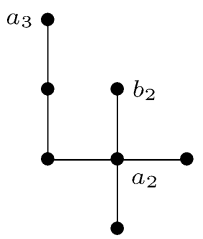

The tree $T_{1}$

We have that $a_{2}$ dominates $b_{2}$ in $\overline{T_{1}}$ and $\left(\overline{T_{1}}: a_{2}\right)$ is the edge ideal of the graph $T_{2}$ :

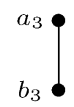

The tree $T_{2}$

Now $a_{3}$ dominates $b_{3}$ in $\overline{T_{2}}$, and the ideal $\left(\overline{T_{2}}: a_{3}\right)$ is the edge ideal of the empty tree. Thus $R(\bar{T}) \simeq \Sigma^{3}\left(S^{-1}\right) \simeq S^{2}$. Note that the independent domination number and the domination number of $T$ are both equal to 3 , as predicted by Corollary 5.5. 

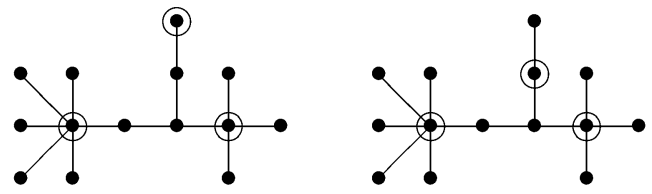

The dominating sets of minimum cardinality

The following corollary of Theorem 4.5 gives an explicit generator of the reduced homology of the independence complex $R(\bar{F})$ of a forest $F$.

Corollary 5.8 Let $F$ be a forest such that $\bar{F}$ is spherical, let $A=\left(a_{1}, \ldots, a_{r}\right)$ be $a$ maximal resolution of $\bar{F}$, and suppose that $a_{i}$ dominates $b_{i}$ in $\left(\bar{F}: a_{1} \cdots a_{i-1}\right)$. Then there is an order of the variables such that $a_{1}<b_{1}<a_{2}<b_{2}<\cdots<a_{r}<b_{r}$ are the first $2 r$ variables; with such an order, the reduced homology of $R(\bar{F})$ is generated by the cycle

$$
z:=\prod_{i=1}^{r}\left(a_{i}-b_{i}\right)
$$

Proof The core $c(A)$ is the ideal generated by $X$. Hence a homology generator of $R(c(A))$ is the class associated to the cycle 1 (in degree -1$)$. Since $a_{i}, b_{i} \in(\bar{F}$ : $a_{1} \cdots a_{i}$ ) for all $i$, it follows that all the variables $a_{i}$ and $b_{i}$ are distinct. Thus there exists an order of the variables such that $a_{1}<b_{1}<a_{2}<b_{2}<\cdots<a_{r}<b_{r}$ are the first $2 r$ variables, and the result follows by Theorem 4.5 .

The cycle $z$ of Corollary 5.8 is a cross-cycle in the sense of [18]. Assuming that the homotopy type of $\bar{F}$ is a sphere, Corollary 5.8 can be proved also using Proposition 3.1 of [18].

\section{The Dominance Complex}

In this section we apply the techniques that we developed in Sections 3 and 4 to the dominance complex of a forest.

Let $G$ be a graph with vertices $x_{1}, \ldots, x_{n}$. Let $G^{\star}$ be the ideal generated by $\left\{\prod_{x \in N\left[x_{i}\right]} x\right\}_{i=1}^{n}$ and $x_{1}^{2}, \ldots, x_{n}^{2}$. The ideal $G^{\star}$ is called the star ideal of $G$, and the simplicial complex $R\left(G^{\star}\right)$ is called the dominance complex of $G$. The faces of the dominance complex of $G$ are the complements of the dominating sets of $G$. The dominance complex of $G$ is never a cone, since every variable divides some minimal generator of $G^{\star}$.

Let $a \in X$; we have

$$
\left(G^{\star}: a\right)=\left((G \backslash\{\text { edges containing } a\})^{\star}, \prod_{y \in N[a] \backslash\{a\}} y\right) .
$$


If $a$ dominates $b$, then $b$ is adjacent to $a$, and all vertices adjacent to $b$ are also adjacent to $a$, i.e., $N[b] \subset N[a]$. Hence if $a$ dominates $b$, we have $\left(G^{\star}: a\right)=(G \backslash\{a\})^{\star}$. Thus, when $F$ is a forest, $F^{\star}$ is always spherical and simple, since the vertex adjacent to a leaf dominates the leaf; this is the unique way a vertex may dominate another one in a forest. The following theorem relates the dominance complex of a forest $F$ to the matching number $\beta_{1}(F)$ and the vertex covering number $\alpha_{0}(F)$, which are known to be equal (see Theorem 2.1).

Theorem 6.1 Let $F$ be a forest; then

(1) $F^{\star}$ is simple;

(2) the dominance complex of $F$ collapses onto the boundary of a cross-polytope of dimension $d\left(F^{\star}\right)$;

(3) $\beta_{1}(F)=\alpha_{0}(F)=d\left(F^{\star}\right)$.

Proof (1) Follows from the remarks preceding the statement of the theorem.

(2) Follows from Theorem 4.3, since if $a$ dominates $b$ in $I$, then $b$ is a leaf and $a b=N[b] \in I$.

(3) Let $\left(a_{1}, \ldots, a_{r}\right)$ be a maximal resolution of $F$, and suppose that $a_{i}$ dominates $b_{i}$ for $i \in[r]$. Clearly $\left\{\left\{a_{1}, b_{1}\right\}, \ldots,\left\{a_{r}, b_{r}\right\}\right\}$ is a matching of $F$, and $\left\{a_{1}, \ldots, a_{r}\right\}$ is a vertex cover of $F$. Since for any graph, the size of a matching is always at most the size of a vertex cover, the result follows.

Remark 6.2 As shown in $[8,14,15]$, the matching number of a forest $F$ can be computed by linear algorithms. Hence there are efficient ways to compute the dimension of the sphere associated to the dominance complex of $F$.

A consequence of Theorem 6.1 is that the removal of a single vertex of $F$ decreases the depth of $F^{\star}$ by at most one: given any matching $M$ of maximum size of $F$, removing a vertex forces the removal of at most one edge from $M$.

Example 6.3 Consider the following tree $T$.

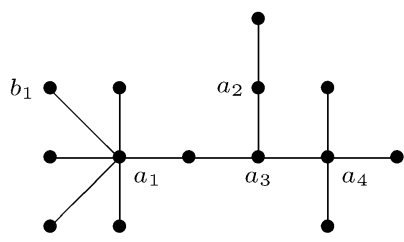

The tree $T$

Let us check that the sequence of vertices $\left(a_{1}, a_{2}, a_{3}, a_{4}\right)$ is a resolution of $T^{\star}$. First of all, the vertex $a_{1}$ dominates $b_{1}$ (and each of the other four leaves adjacent to $a_{1}$ ). The ideal $\left(T^{\star}: a_{1}\right)$ is the star ideal of the graph $T_{1}=T \backslash$ edges containing $\left.a_{1}\right\}$ : 


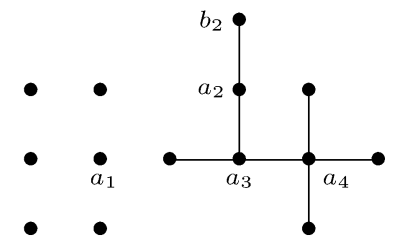

The forest $T_{1}$

Note that the simplicial complexes associated to $T_{1}^{\star}$ and to the star ideal of the graph $T_{1} \backslash$ isolated vertices of $\left.T_{1}\right\}$ are the same; thus, in what follows, we always remove isolated vertices. We have that $a_{2}$ dominates $b_{2}$ in $T_{1}^{\star}$ and $\left(T_{1}^{\star}: a_{2}\right)$ is the star ideal of the graph $T_{2}$ :

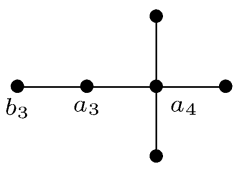

The tree $T_{2}$

Now $a_{3}$ dominates $b_{3}$ in $T_{2}^{\star}$, and the ideal $\left(T_{2}^{\star}: a_{3}\right)$ is the star ideal of the tree $T_{3}$ :

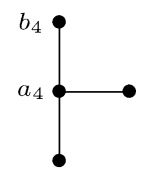

The tree $T_{3}$

Finally, $a_{4}$ dominates $b_{4}$ in $T_{3}^{\star}$, and the ideal $\left(T_{3}^{\star}: a_{4}\right)$ is the star ideal of the empty graph. Thus $R\left(T^{\star}\right) \simeq \Sigma^{4}\left(S^{-1}\right) \simeq S^{3}$. Note that the matching number and the vertex covering number of $T$ are both equal to 4 , as predicted by Theorem 6.1.
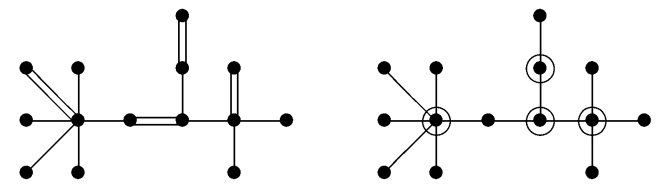

A matching and a vertex cover of maximum cardinality

We state a corollary of Theorem 4.5 corresponding to the result of Corollary 5.8 about the independence complex. Note that despite the substantial difference between the dominance complex and the independence complex, the statements of Corollaries 5.8 and 6.4 are completely analogous. The machinery of the core allows for an identical proof of the two results. Therefore, here we omit the proof. 
Corollary 6.4 Let $F$ be a forest and $A=\left(a_{1}, \ldots, a_{r}\right)$ be a maximal resolution of $F^{\star}$, and suppose that $a_{i}$ dominates $b_{i}$ in $\left(F^{\star}: a_{1} \cdots a_{i-1}\right)$. Then there is an order of the variables such that $a_{1}<b_{1}<a_{2}<b_{2}<\cdots<a_{r}<b_{r}$ are the first $2 r$ variables; with such an order, the reduced homology of $R\left(F^{\star}\right)$ is generated by the cycle

$$
z:=\prod_{i=1}^{r}\left(a_{i}-b_{i}\right) .
$$

Note that $F \backslash\left\{a_{1}, \ldots, a_{r}\right\}$ has no edges and the complementary of $\left\{a_{1}, \ldots, a_{r}\right\}$ is a maximal face of $R\left(F^{\star}\right)$. Hence the cycle $z$ of Corollary 6.4 is a cross-cycle in the sense of [18].

Remark 6.5 Clearly, if $F$ has no isolated vertices, then $\gamma(F) \leq \alpha_{0}(F)$. Thus $d(\bar{F}) \leq d\left(F^{\star}\right)$. In particular, if $\bar{F}$ is spherical, then the sphere associated to the independence complex has dimension at most the dimension of the sphere associated to the dominance complex.

Acknowledgements We would like to thank the anonymous referees for the very careful reading of the paper and for precise and helpful comments that improved the clarity of the text. We would like to thank also S. Hedetniemi for pointing us to some references on algorithms for computing invariants of graphs.

The second author wishes to express his gratitude to Università "La Sapienza", Rome, where this research was carried out.

\section{References}

1. Allan, R.B., Laskar, R.: On domination and independent domination numbers of a graph. Discrete Math. 23, 73-76 (1978)

2. Allan, R.B., Laskar, R., Hedetniemi, S.: A note on total domination. Discrete Math. 49, 7-13 (1984)

3. Beyer, T., Hedetniemi, S., Mitchell, S., Proskurowski, A.: Independent domination in trees. In: Proceedings of the Eighth Southeastern Conference on Combinatorics, Graph Theory and Computing, Louisiana State Univ., Baton Rouge, LA, 1977. Congressus Numerantium, vol. XIX, pp. 321-328. Utilitas Math., Winnipeg (1977)

4. Bollobás, B.: Modern Graph Theory. Graduate Texts in Mathematics, vol. 184. Springer, Berlin (1998)

5. Bollobás, B., Cockayne, E.J.: Graph-theoretic parameters concerning domination, independence, and irredundance. J. Graph Theory 3(3), 241-249 (1979)

6. Bousquet-Mélou, M., Linusson, S., Nevo, E.: On the independence complex of square grids. arXiv:math/0701890v2 [math.CO]

7. Cockayne, E.J., Goodman, S.E., Hedetniemi, S.T.: A linear algorithm for the domination number of a tree. Inf. Process. Lett. 4, 41-44 (1975)

8. Mitchell, S., Cockayne, E., Hedetniemi, S.: Linear algorithms on recursive representations of trees. J. Comput. Syst. Sci. 18(1), 76-85 (1979)

9. Cohen, M.M.: A Course in Simple-Homotopy Theory. Graduate Texts in Mathematics, vol. 10. Springer, New York (1973)

10. Diestel, R.: Graph Theory. Graduate Texts in Mathematics, vol. 173. Springer, Berlin (1997)

11. Engström, A.: Complexes of directed trees and independence complexes. arXiv:math/0508148v1 [math.CO]

12. Ehrenborg, R., Hetyei, G.: The topology of the independence complex. Eur. J. Comb. 27(6), 906-923 (2006)

13. Erdős, P., Tuza, Z.: Vertex coverings of the edge set in a connected graph. In: Graph Theory, Combinatorics, and Algorithms, Kalamazoo, MI, 1992, vols. 1, 2, pp. 1179-1187. Wiley-Interscience, New York (1995) 
14. Goodman, S., Hedetniemi, S., Mitchell, S.: Some linear algorithms on trees. In Proceedings of the Sixth Southeastern Conference on Combinatorics, Graph Theory, and Computing, Florida Atlantic Univ., Boca Raton, FL, 1975. Congressus Numerantium, vol. XIV, pp. 467-483. Utilitas Math., Winnipeg (1975)

15. Goodman, S., Hedetniemi, S., Tarjan, R.E.: $b$-matchings in trees. SIAM J. Comput. 5(1), 104-108 (1976)

16. Haynes, T.W., Hedetniemi, S.T., Slater, P.J.: Fundamentals of Domination in Graphs. Monographs and Textbooks in Pure and Applied Mathematics, vol. 208. Marcel Dekker, New York (1998)

17. Henning, M.A., Yeo, A.: Total domination and matching numbers in claw-free graphs. Electron. J. Comb. 13(1), 59 (2006)

18. Jonsson, J.: Certain homology cycles of the independence complex of grid graphs. Preprint

19. Klivans, C.: Threshold graphs, shifted complexes, and graphical complexes. arXiv:math/0703114v1 [math.CO]

20. Kozlov, D.: Complexes of directed trees. J. Comb. Theory Ser. A 88(1), 112-122 (1999)

21. Kozlov, D.: Directed trees in a string, real polynomials with triple roots, and chain mails. Discrete Comput. Geom. 32(3), 373-382 (2004)

22. Laskar, R., Walikar, H.B.: On domination related concepts in graph theory. In: Combinatorics and Graph Theory. Lecture Note in Mathematics, vol. 885, pp. 308-320. Springer, Berlin (1981)

23. Miller, E., Sturmfels, B.: Combinatorial Commutative Algebra. Graduate Texts in Mathematics, vol. 227. Springer, New York (2005)

24. Munkres, J.R.: Elements of Algebraic Topology. Perseus Books, London (1984)

25. Mynhardt, C.M.: Vertices contained in every minimum dominating set of a tree. J. Graph Theory 31(3), 163-177 (1999)

26. Stanley, R.: Combinatorics and Commutative Algebra, 2nd edn. Progress in Mathematics, vol. 41. Birkhäuser, Basel (1996)

27. Wassmer, A.: A dual independence complex. PhD thesis, Technische Universität Berlin (2005) 